\title{
Erratum to: Wireless three-hop networks with stealing II: exact solutions through boundary value problems
}

\author{
Fabrice Guillemin - Charles Knessl • \\ Johan S. H. van Leeuwaarden
}

Published online: 28 August 2014

(C) Springer Science+Business Media New York 2014

\section{Erratum to: Queueing Syst (2013) 74:235-272 DOI 10.1007/s11134-012-9332-8}

Contrary to what we claimed in [5], the solution to the Riemann-Hilbert problem (4) considered in [5] for some domain $D_{x}$ is in general not the restriction to $D_{x}$ of the solution to the modified Riemann-Hilbert problem (6) in [5]. This occurs only when $D_{x}$ is a circle, which is not the case considered in that paper. To solve problem (4), we have to consider the conformal mapping $\gamma_{x}(x)$ from $D_{x}$ onto the unit disk $D$. We are thus led to consider a modified version of the Riemann-Hilbert problem (5) formulated in that paper.

The online version of the original article can be found under doi:10.1007/s11134-012-9332-8.

\section{F. Guillemin}

Orange Labs, 2 Avenue Pierre Marzin, 22300 Lannion, France

e-mail: Fabrice.Guillemin@orange-ftgroup.com

C. Knessl

Department of Mathematics, Statistics and Computer Science, University of Illinois at Chicago, 815 South Morgan Street, Chicago, IL 60607-7045, USA

e-mail: kness1@uic.edu

J. S. H. van Leeuwaarden $(\varangle)$

Department of Mathematics and Computer Science, Eindhoven University of Technology, Room HG 9.13, P.O. Box 513, 5600 MB Eindhoven, The Netherlands e-mail: j.s.h.v.leeuwaarden@tue.nl 


\section{Modified boundary value problem}

To compute the function $P(x, 0)$, by using arguments similar to those in [5], we consider the function $P_{x}(x)$ given by Eq. (20) in [5], namely

$$
P_{x}(x)=P(x, 0)-\frac{R_{x}}{x-x^{*}}+P(0,0)
$$

The function $P_{x}(x)$ is analytic in $D_{x}$ and satisfies for $x \in \partial D_{x}$ and $y=Y^{*}(x)$

$$
\Re\left(i \frac{h_{2}(x, y)}{h_{3}(x, y)} P_{x}(x)\right)=\mathfrak{s}\left(\frac{R_{x} h_{2}(x, y)}{h_{3}(x, y)\left(x-x^{*}\right)}\right) .
$$

To solve this problem, we consider the conformal mapping $\gamma_{x}(x)$ from $D_{x}$ onto the unit disk. This conformal mapping can be chosen to preserve the symmetry with respect to the horizontal axis and to satisfy $\gamma_{x}\left(X^{+}\left(y_{1}\right)\right)=-1$ and $\gamma_{x}\left(X^{+}\left(y_{2}\right)\right)=1$. Moreover, by imposing the condition $\gamma_{x}(0)=0$, the conformal mapping $\gamma_{x}(x)$ is unique.

We are then led to consider the following problem on the unit circle: The function $P_{x}\left(c_{x}(u)\right)$ is analytic in the unit disk $D$ and satisfies for $u$ on the unit circle $C$

$\Re\left(i \frac{h_{2}\left(c_{x}(u), Y^{*}\left(c_{x}(u)\right)\right)}{h_{3}\left(c_{x}(u), Y^{*}\left(c_{x}(u)\right)\right)} P_{x}\left(c_{x}(u)\right)\right)=\mathfrak{s}\left(\frac{R_{x} h_{2}\left(c_{x}(u), Y^{*}\left(c_{x}(u)\right)\right)}{h_{3}\left(c_{x}(u), Y^{*}\left(c_{x}(u)\right)\right)\left(c_{x}(u)-x^{*}\right)}\right)$,

where $c_{x}(u)$ is the inverse of $\gamma_{x}(x)$ and maps the unit disk onto the domain $D_{x}$.

The above Riemann-Hilbert problem is of the following form: Find a function which is analytic in $D$, continuous in the closure $\bar{D}$ of the unit disk, and satisfies

$$
\Re(i a(u) f(u))=c(u) .
$$

Since, by construction, the function $f(u)$ is real on the real axis, it is possible to define by the reflection principle [3] the function $g(u)=\overline{f(1 / \bar{u})}$, which is analytic in $\mathbb{C} \backslash \bar{D}$. The function $f(u)$ (resp. $g(u)$ ) is the restriction to $D$ (resp. $\mathbb{C} \backslash \bar{D}$ ) of the sectionally analytic function $F(u)$, which satisfies the following Riemann-Hilbert problem: Find a sectionally analytic function $F(u)$ with respect to the unit circle, bounded at infinity $(F(\infty)=f(0))$ and such that for $u \in C$

$$
a(u) F^{i}(u)-\overline{a(u)} F^{e}(u)=-2 i c(u),
$$

where $F^{i}(u)\left(\right.$ resp. $\left.F^{e}(u)\right)$ is the interior (resp. exterior) limit of the function $F(u)$ on the unit circle.

The solution to this problem, when it exists, is given by [2]

$$
F(u)=\frac{\phi^{i}(u)}{2 i \pi} \int_{C} \frac{C(z)}{\phi^{(i)}(z)(z-u)} d z+Q(u) \phi(u),
$$


where $Q(u)$ is a polynomial, which can be determined by using the conditions at infinity, the function $C(u)$ is given by

$$
C(u)=-2 i \frac{c(u)}{a(u)},
$$

and the function $\phi(u)$ is defined by

$$
\phi(u)=\left\{\begin{array}{l}
\exp \left(\frac{1}{2 i \pi} \int_{C} \log \left(z^{-\kappa} \frac{\overline{a(z)}}{a(z)}\right) \frac{d z}{z-u}\right), \quad u \in D, \\
\frac{1}{u^{\kappa}} \exp \left(\frac{1}{2 i \pi} \int_{C} \log \left(z^{-\kappa} \frac{\overline{a(z)}}{a(z)}\right) \frac{d z}{z-u}\right), u \in \mathbb{C} \backslash \bar{D},
\end{array}\right.
$$

with $\kappa$ denoting the index of the Riemann-Hilbert problem and $\phi^{(i)}(u)$ being the interior limit of the function $\phi(u)$ on the unit circle. When $\kappa<0$, the solution to the Riemann-Hilbert problem exists and is unique if and only if for $k=0, \ldots,|\kappa|-1$

$$
\int_{C} \frac{z^{k} C(z)}{\phi^{(i)}(z)} d z=0 ;
$$

in that case, the polynomial $P(u) \equiv 0$. When $\kappa=0$, the solution is unique and $P(u)$ is a constant.

\section{Conformal map}

We determine in this section the conformal mapping $\gamma_{x}(x)$ from the domain $D_{x}$ onto the unit disk.

Let $w_{x}(x)$ be the conformal gluing function for the shift transformation $t_{x}(x)$ and the contour $\partial D_{x}$, where

$$
t_{x}(x) \stackrel{\text { def }}{=} \bar{x}=\frac{Y^{*}(x)}{(1-p) x}
$$

The function $w_{x}(x)$ is solution to the Carleman problem

$$
w_{x}\left(t_{x}(x)\right)=w_{x}(x)
$$

for $x \in \partial D_{x}$. As proved in [8], the function $w_{x}(x)$ conformally transforms the domain $D_{x}$ onto the complex plane deprived of the positively oriented $\operatorname{arc} L_{x}$ joining the points $w_{x}\left(X^{+}\left(y_{2}\right)\right)$ and $w_{x}\left(X^{+}\left(y_{1}\right)\right)$. 
For the case under consideration, the conformal gluing function $w_{x}(t)$ is given in [1] and [7, Remark 16] by

$$
w_{x}(t)=\frac{t}{\left(t-x_{2}\right)\left(t-\sqrt{\frac{1+p}{(1-p)^{2} x_{2}}}\right)^{2}} .
$$

It is worth noting that $w_{x}(x)$ is real for $x \in \partial D_{x}$ and that

$$
w_{x}\left(X^{+}\left(y_{1}\right)\right)<w_{x}\left(X^{+}\left(y_{2}\right)\right) .
$$

Using the fact that for reals $a<b$ the function $v(a, b ; x)$ defined by

$$
v(a, b ; x)=\frac{\sqrt{\frac{x-a}{x-b}}-1}{\sqrt{\frac{x-a}{x-b}}+1}
$$

conformally maps the complex plane deprived of the segment $[a, b]$ onto the unit disk, the conformal mapping $\gamma_{x}(x)$ is given by

$$
\gamma_{x}(x)=v\left(w_{x}\left(X^{+}\left(y_{1}\right)\right), w_{x}\left(X^{+}\left(y_{2}\right)\right) ; w_{x}(x)\right)
$$

The function $w_{x}(x)$ takes value in $\left[w_{x}\left(X^{+}\left(y_{1}\right)\right), w_{x}\left(X^{+}\left(y_{2}\right)\right)\right]$ for $x \in\left[x_{3}, x_{4}\right]$. It can then be shown that the equation with degree three $w_{x}(t)=w_{x}(x)$ for $x \in$ $\left[x_{3}, x_{4}\right]$ has three solutions, namely $x, Y^{*}(x) /((1-p) x)$ and $Y_{*}(x) /((1-p) x)$. Since $Y^{ \pm}\left(\left[x_{3}, x_{4}\right]\right)=\partial D_{x}$, we deduce that the conformal mapping $\gamma_{x}(x)$ can be analytically continued in the whole of $\mathbb{C} \backslash\left[x_{3}, x_{4}\right]$ with an algebraic singularity at point $x_{3}$.

\section{Solution of the corrected boundary value problem for $P(x, 0)$}

By using the above results, Eq. (34) in [5] should then read for $x \in D_{x}$ (when $p \leq p^{*}$, the index $\kappa_{x}=0$ )

$$
\varphi_{x}^{(1)}(x)=\exp \left(\frac{1}{2 i \pi} \int_{\partial D_{x}} \frac{\log \left(A_{x}(z)\right)}{z-x} k_{x}(x, z) d z\right),
$$

where the kernel $k_{x}(x, z)$ is given by

$$
k_{x}(x, z)=\frac{(z-x) \gamma_{x}^{\prime}(z)}{\gamma_{x}(z)-\gamma_{x}(x)}
$$

The kernel $k_{x}(x, z)$ is defined for $x \in D_{x}$ and when $x=z \in D_{x}, k_{x}(x, z)=1$. 
The function $\varphi_{x}^{(1)}(x)$ can be continued by setting

$$
\begin{aligned}
& \varphi_{x}^{(1)}(x)= \\
& \left\{\begin{array}{l}
\exp \left(\frac{1}{2 i \pi} \int_{\partial D_{x}} \frac{\log \left(A_{x}(z)\right)}{z-x} k_{x}(x, z) d z\right), \text { for } x \in D_{x}, \\
A_{x}(x) \exp \left(\frac{1}{2 i \pi} \int_{\partial D_{x}} \frac{\log \left(A_{x}(z)\right)}{z-x} k_{x}(x, z) d z\right), \text { for } x \in{\overline{D_{x}}}^{c} \cap V_{x} \cap D\left(0, x_{3}\right),
\end{array}\right.
\end{aligned}
$$

where $V_{x}$ is the domain of analyticity of the function $\log \left(A_{x}(x)\right)$.

Similarly, Eq. (37) in [5] should read

$$
\begin{aligned}
& \varphi_{x}^{(2)}(x)= \\
& \exp \left(\frac{1}{2 i \pi} \int_{\mathcal{C}_{x}} \frac{\log \left(\mathcal{A}_{x}(z)\right)}{z-x} k_{x}(x, z) d z\right), \text { for } x \in \overline{D_{x}} \\
& \mathcal{A}_{x}(x) \exp \left(\frac{1}{2 i \pi} \int_{\partial D_{x}} \frac{\log \left(\mathcal{A}_{x}(z)\right)}{z-x} k_{x}(x, z) d z\right), \text { for } x \in{\overline{D_{x}}}^{c} \cap \mathcal{V}_{x} \cap D\left(0, x_{3}\right) .
\end{aligned}
$$

Eq. (35) is erroneous.

The function $P(x, 0)$ is eventually given by

$$
\begin{aligned}
P(x, 0)= & \frac{R_{x}}{x-x^{*}}+B_{x}(x)+\frac{\varphi_{x}(x)}{2 i \pi} \int_{\partial D_{x}} \frac{B_{x}(z)-B_{x}(x)}{\phi_{x}^{i}(z)(z-x)} k_{x}(x, y) d z \\
& -P(0,0)+K_{x} \varphi_{x}(x),
\end{aligned}
$$

where

$$
\varphi_{x}(x)= \begin{cases}\varphi_{x}^{(1)}(x), & \text { when } p \leq p^{*}, \\ \varphi_{x}^{(2)}(x), & \text { when } p>p^{*}\end{cases}
$$

with $\varphi_{x}^{(1)}(x)$ as in (3) and $\varphi_{x}^{(2)}(x)$ as in (4), $K_{x}$ is some constant, and the function $B_{x}(x)$ defined by Eq. (31) in [5].

\section{Solution of the corrected boundary value problem for $P(0, y)$}

For determining the function $P(0, y)$, the conformal mapping $\gamma_{y}(y)$ from the domain $D_{y}$ onto the unit disk is required, which is given by (see $\left.[1,10]\right)$

$$
\gamma_{y}(y)=\frac{y k_{y}\left(\frac{\eta}{1+p}\right)-(1+p) \eta k_{y}\left(\frac{y}{1+p}\right)}{y k_{y}\left(\frac{\eta}{1+p}\right)+(1+p) \eta k_{y}\left(\frac{y}{1+p}\right)}
$$


where

$$
k_{y}(y)=(\alpha-y) \sqrt{\frac{1-p}{1+p}-(1-p)^{2} \alpha^{2} y}
$$

with $\alpha=Y^{*}\left(x_{2}\right) /(1+p)$ and $\eta$ is any constant in $(0, \alpha)$. In the following, we take $\eta=\alpha / 2$. By using similar arguments as those for the conformal mapping $\gamma_{x}(x)$, we can prove that the conformal mapping $\gamma_{y}(y)$ is analytic in $D\left(0, y_{3}\right)$ with continuous limits on the boundary.

Equation (51) in [5] should read

$$
\phi_{y}(y)=\exp \left(\frac{1}{2 i \pi} \int_{\partial D_{y}} \frac{\log \left(A_{y}(z)\right)}{z-y} k_{y}(y, z) d z\right)
$$

where the kernel $k_{y}(y, z)$ is defined by

$$
k_{y}(y, z)=\frac{(z-y) \gamma_{y}^{\prime}(z)}{\gamma_{y}(z)-\gamma_{y}(y)}
$$

The interior limit of $\phi_{y}(y)$ on the contour $\partial D_{y}$ is defined by

$$
\phi_{y}^{(i)}(y)=\exp \left(\frac{1}{2} \log \left(A_{y}(y)\right)+\frac{1}{2 i \pi} \oint_{\partial D_{y}} \frac{\log \left(A_{y}(z)\right)}{z-y} k_{y}(y, z) d z\right) .
$$

The restriction to $D_{y}$ of the function $\phi_{y}(y)$ coincides with the restriction to $D_{y}$ of the function $\varphi_{y}(y)$ defined by

$$
\varphi_{y}(y)= \begin{cases}\phi_{y}(y), & \text { if } y \in D_{y}, \\ A_{y}(y) \phi_{y}(y), & \text { if } y \notin D_{y},\end{cases}
$$

which is a meromorphic function in $\mathbb{C} \backslash\left[y_{3}, \infty\right)$ with a pole at the point $y^{* *}$.

The function $P(0, y)$ is given in $\mathbb{C} \backslash\left[y_{3}, y_{4}\right]$ by

$$
\begin{aligned}
P(0, y)= & \frac{R_{y}}{y-y^{* *}}-P(0,0)+B_{y}(y)+\frac{\varphi_{y}(y)}{2 i \pi} \int_{\partial D_{y}} \frac{B_{y}(z)-B_{y}(y)}{\phi_{y}^{i}(z)(z-y)} k_{y}(z, y) d z \\
& +K_{y} \varphi_{y}(y)
\end{aligned}
$$

where $B_{y}(y)$ is given by Eq. (50) in [5] and $K_{y}$ is a constant as in Eq. (56) in [5].

\section{Asymptotic analysis}

In spite of these modifications, the asymptotic analysis given in Sect. 6 of [5] is correct; the factors $\kappa_{1}$ and $\kappa_{2}$ should be computed by using the new formulas giving $P\left(0, y^{*}\right)$ and $P(0,0)$ depending on the conformal mapping $\gamma_{y}(y)$. 
The same flaw occurs in [6] but the asymptotic analysis is also correct.

\section{References}

1. Blanc, J.P.C.: The relaxation time of two queues systems in series. Commun. Statist. Stochastic Models 1, 1-16 (1985)

2. Dautray, R., Lions, J.L.: Analyse Mathématique et Calcul Numérique pour les Sciences et les Techniques. Masson, Paris (1995)

3. Dieudonne, J.: Calcul Infinitésimal. Hermann, Paris (1980)

4. Fayolle, G., Iasnogorodski, R., Malyshev, V.: Random Walks in the Quarter Plane. Springer-Verlag, New York (1999)

5. Guillemin, F., van Leeuwaarden, J.S.H.: Rare event asymptotic for a random walk in the quarter plane. Queueing Syst. 67, 1-32 (2011)

6. Guillemin, F., Knessl, C., van Leeuwaarden, J.S.H.: Wireless three-hop networks with stealing II: exact solutions through boundary value problems. Queueing Syst. 74, 235-272 (2013)

7. Kurkova, I., Raschel, K.: Random walks in $\mathbb{Z}_{+}^{2}$ with non-zero drift absorbed at the axes. Bull. Soc. Math. France 139, 341-387 (2011)

8. Litvinchuk, G.: Solvability of Boundary Value Problems and Singular Integral Equations with Shift. Springer, Dordrecht (2000)

9. Muschelischwili, N.I.: Singuläre Integralgleichungen. Akademie Verlag, Berlin (1965)

10. van Leeuwaarden, J. S. H., Resing, J. A. C.: A tandem queue with coupled processors: computational issues. Queueing Syst. 51(1-2), 29-52 (2005) 